\title{
Timesharing on the PDP-11 under RT-11
}

\author{
KARL W. SCHOLZ \\ The Medical College of Pennsylvania, Division of Social Science, The Department of Psychiatry, \\ Philadelphia, Pennsylvania 19129
}

\begin{abstract}
A timesharing system for the Digital Equipment Corporation PDP-11 is described. In keeping with the general philosophy of the RT-11 operating system, the permanently resident portion of the system is kept to an absolute minimum size. The system supports from 2 to 16 users, and provides each with full editing and batch execution capabilities.
\end{abstract}

A majority of the users of the Digital Equipment Corporation PDP-11 in scientific and academic environments have chosen the RT-11 operating system as the system which offers the greatest range of facilities with the smallest resident memory requirements of any Digital systems. However, despite its versatility, RT-11 is inherently a single-user (with the exception of the Multiple User Basic option) and, as such, much potentially valuable computer time is wasted. The present paper describes the design and implementation of a multiple-access version of RT- 11 which extends the full power of RT to a community of up to 16 simultaneous users. Our system, MART (for Multiple Access Real Time), has been designed to maximize ease of operation with a minimal resident memory requirement (less than 1,700 words plus 611 words per user).

RT-11 is designed as a two-partition (foregroundbackground) system. The MART resident monitor executes as a foreground job which supervises the allocation of the background partition to various users. The design provides each simultaneous user the benefits of a fully interactive text editor (a subset of the standard RT editor), a Concise Command Language (CCL) for system resource management, a facility for the interactive execution of specially prepared foreground programs, and job queue management for the batch execution of FORTRAN, BASIC, or MACRO (Digital's assembly language) programs. Complete accounting facilities are provided which retain total log-on time and total batch execution time for each user. MART permits several users to engage in real-time data acquisition at the same time others are editing source files or data files and still others are executing large batch programs for data reduction or statistical analysis.

\section{SYSTEM COMPONENTS}

MART consists of four primary components: a monitor, a collection of port tables, a set of nonresident overlays, and a job queue manager.

1. The monitor, which is permanently resident in the foreground, manages the operation of the entire system. The monitor contains a dispatcher to determine which user to service at any point in time, an overlay manager to load and execute the nonresident overlays, and a collection of routines to handle input/output and interactive text editing.

2. At the time the system is generated, one port table is allocated for each potentially active port on the system. Each port table contains a collection of flags and a buffer which is used as either an edit buffer or as space for the execution of nonresident overlays.

3. All utility functions, such as file creation and deletion, disk directory manipulation, and job queue insertion are implemented as nonresident overlays. The overlays, which are written in position-independent code, are loaded as needed into the port table of the user requiring the service.

4. Job management is supervised in part by routines in the resident monitor, and in part by a nonresident program which executes in the background. Whenever this program detects that a foreground overlay has inserted a job into the job queue, the background job manager replaces itself with the indicated job which is then free to run to completion. Upon job completion, the job manager is reloaded, at which time it performs postjob cleanup. If additional jobs remain in the queue, they are similarly executed; if not, the job manager idles.

\section{SYSTEM OPERATION}

In operation, MART is table driven. During periods of nonactivity the dispatcher in the foreground monitor idles awaiting the setting of "I/O complete" flags in each port table. Each time an $1 / O$ operation-complete interrupt occurs, simple interrupt service routines set these flags. As soon as the dispatcher detects a set flag, it points a processor general register to the port table of the appropriate user. All address references in the remainder of the monitor are expressed as offsets with respect to this register. The dispatcher then transfers control to the monitor code that interrogates "service required" flags in the port table to determine in what activity the associated user was engaged. Service for that user then continues until he is next $I / O$ bound (e.g., awaiting completion of an input/output operation), at which time control returns to the dispatcher. The dispatcher 
then continues polling the $I / O$ complete flags, and either transfers control to service a set flag or idles if no flags are set.

In our implementation, both the $\mathrm{I} / \mathrm{O}$ complete and the service required flags for each port are grouped together as 161 -bit flags in the first word of each port table. Thus, the dispatcher idle consists of sequentially inspecting this single flag word in each port table. If the inspection of all flag words (there will be $\mathrm{n}$ flag words on a system configured for $\mathbf{n}$ simultaneous users) indicates that no activity is required, foreground activity is suspended. In operation, each interrupt service routine sets the appropriate $\mathrm{I} / \mathrm{O}$ complete flag, posts this event to the suspended foreground mainline (the dispatcher), and then clears the interrupt and exits. Thus, each time an event is posted, the dispatcher awakens and initiates the polling sequence described above.

In summary, this method of timesharing allows activity for all users to take place at a single priority which is lower than the priority at which any $I / O$ interrupt activity takes place. In addition to its ease of implementation (the nucleus of the dispatcher is only 20 instructions long), this method permits the equitable distribution of time among all simultaneous users without the necessity of more complex time-slicing or queue-driven algorithms. This system design is described in more detail in Scholz (1972).

\section{INTERACTIVE COMMAND EXECUTION AND TEXT EDITING}

The remainder of the foreground resident system consists of facilities for overlay management and for the supervision of interactive text editing. Each port table contains a buffer which is two disk blocks ( 1,024 bytes) in length. Whenever a user is engaged in text editing, this buffer is used as a window through which the file under modification is scrolled. Once the user terminates the editing process, the buffer is flushed to the disk and the modified file is closed. Thus, whenever editing is not in progress, each user's buffer is available for the execution of code. In order to take advantage of this fact, all those MART utility functions which are not intimately involved with interactive editing are implemented as overlays, each of which is a stand-alone position-independent program capable of being executed from any port table buffer.

MART's command syntax requires each utility command to be preceded by a period. (Thus, utility commands are syntactically distinct from edit commands, which do not begin with a period.) Whenever the resident monitor identifies a period as the first character in an input line, a master overlay is loaded into the port table for the user that entered the command. The master overlay parses the remainder of the input line, and if it recognizes a valid command, it chains to the appropriate overlay to service the command.
Thus, the MART command repertoire is readily extensible: Adding new commands requires only modifying tables in the master overlay and preparing a new overlay to implement the new command. It should be emphasized that adding additional commands in no way alters the size of the resident code. Currently, MART's Concise Command Language (CCL) includes 14 commands for frequently used operations (such as LOGON, LOGOFF, COPY, DELETE, RUN, etc.).

Any command that is not preceded by a period is interpreted as an edit command. The editor has been designed to resemble the standard RT-11 editor as closely as possible (only edit macros and save/unsave options are omitted from the MART editor). Since the port table buffers are utilized by the editor as edit buffers, they are unavailable for overlay execution while editing is in progress. For this reason, the code for the editor must be resident. All constants required by the editor (such as file pointers, buffer pointers, and mode flags) are stored in the port tables. As indicated above, the dispatcher determines which user's turn it is, and enters the editor code with a register pointing to his port table. Thus, an indefinite number of users can be simultaneously engaged in text editing. Servicing additional users requires only the addition of more port tables, all of which share the single copy of the reentrant editor code.

\section{INTERACTIVE PROGRAM EXECUTION}

So far, we have described MART's facilities for interactive program preparation and batch program execution. Facilities are also provided for the interactive execution of specially prepared programs utilizing the overlay facility described above. Two restrictions must be observed in the preparation of code which is to be executed interactively. First, the program's I/O must be integrated into the dispatcher in MART's resident monitor, and second, the program must be positionindependent since it will be executed from any user's port table. The observation of these restrictions is eased through the use of a carefully designed macro library which permits the creation of MART-compatible interactive programs with a minimum of difficulty. The macro library includes one macro that can be used to chain from program to program. Thus, even though the size of a port table limits the size of an interactive program segment to 2,000 bytes (octal), chaining permits the creation of indefinitely complex interactive programs.

\section{COMMAND SYNTAX ARCHITECTURE}

Although MART provides sophisticated features such as the interactive program execution facility, it was designed primarily for a naive user community. Considerable effort was placed into the selection of 
specific commands and in the syntactic architecture of the user interface. Both feedback from users and the author's experience with other interactive systems (Halff, Scholz, \& Walker, 1975; Scholz \& Holly, 1975) were utilized in the design of the command syntax. Our first choice was the decision to incorporate a Concise Command Language into MART. The availability of a CCL as the vehicle for expression of the user's intent can be contrasted with the general RT philosophy in which a small number of utility programs are provided, each of which has numerable options selected through the use of flags. For example, RT's PIP (an acronym for the Peripheral Interchange Program) is used for a variety of operations such as data transfer, file deletion, and other aspects of disk management. Each option is selected by suffixing the command with a special flag. In MART, each corresponding operation is invoked by a command which specifies the exact operation, for example, COPY, RENAME, PRINT, LIST, DELETE, RUN. In our experience, it has proven far simpler to instruct a newcomer in the use of an operating system if his/her intent can be expressed by entering concise commands, rather than by invoking general procedures which must then be tailored to perform the desired activity.

A second consideration in the design of the system command syntax concerns the issue of user "friendliness." The constraints which characterize command syntax in any computer dialog can be viewed as being either required or optional. In the former case, some syntactic constraints are mandatory in order to avoid ambiguity (such as requiring a space or punctuation after an abbreviated command). In the second case, command syntax often allows the use of punctuation as delimiters that are not required for syntactic disambiguation, but are included for clarification to the user (such as the use of spaces in FORTRAN). In general, systems which tolerate variations in syntax for the sole purpose of the convenience of the user will be viewed as "friendly" systems, while systems that demand rigid adherence to syntactic rules will appear "unfriendly" or even "imposing" to the newcomer. Thus, in the design of MART, we have attempted to promote the feeling of user friendliness by permitting user-determined syntactic variations. For example, the MART editor (syntactically identical to the RT editor for the sake of compatibility) requires that strings being inserted into a file or strings serving as search targets for context editing be terminated by the ASCII ESC delimiter. However, although the remaining edit commands and all the utility commands (the CCL referred to above) are customarily terminated by a carriage return, MART will tolerate ending these commands with an "ESC." Even this simple syntactic liberalization has served to greatly facilitate teaching newcomers to use the system. As a second example, MART, like many systems, permits any command to be either specified in full or abbreviated to just those several letters necessary to syntactically differentiate it from other commands. And third, MART includes a "HELP" facility, where a user can type HELP preceding any command in order to receive a concise description of that command.

\section{IMPLEMENTATION AND TRANSPORTABILITY}

The prototype of MART was developed for, and is now running on, a 56K-byte PDP-11/40 with one tape drive and a 20 megabyte disk (DEC's RPR02). Eight access ports are interfaced using a multiplexer (DEC's DH11), four hard-wired to CRT terminals (DEC's VT55s) and four connected to the phone system using Bell 113B modems. When the entire MART system is loaded, approximately $36 \mathrm{~K}$ bytes are available for batch execution of programs. This space is more than adequate for the execution of statistics package programs and for the development and compilation of BASIC, FORTRAN, and MACRO programs.

In an effort to facilitate transportability, all variable parameters (such as the total number of ports on the system or what model CPU is to host the system) have been entered via equates. Thus, system generation for a new machine is quite simple-first appropriate values for the equates are entered into the source code for MART, then the system is assembled and linked. Once the required device handlers have been prepared, MART is ready to load and put into operation. We have prepared complete documentation on the system, including both user manuals and a detailed description of the system generation procedure.

\section{SUMMARY}

In summary, timesharing under RT is not only relatively simple to implement, but in many ways superior to other approaches to small-system timesharing. Not only are both software and hardware costs greatly reduced, but the power of a well-designed minicomputer is extended to a number of simultaneous users. Our system was delivered in June, and by October we were fully operational, servicing a user community ranging from individuals utilizing only canned statistical programs for data analysis to users programming complex operations in machine language.

\section{REFERENCES}

HalfF, H., Scholz, K, W., \& WAlkeR, J. H. An extension of Kieras' general experimental programming system. Behavior Research Methods \& Instrumentation, 1975, 7, 464-470.

ScHoLz, K. W. Computerized process control in behavioral science research. Behavior Research Methods \& Instrumentation, 1972, 4, 203-208.

Scholz, K. W., \& Holly, B. A 64-station computer-assisted teaching and research facility. Behavior Research Methods \& Instrumentation, 1975, 7, 301-304. 